\title{
Novel spinal instrumentation to enhance osteogenesis and fusion: a preliminary study
}

\author{
Matthew R. MacEwan, PhD, ${ }^{1,3}$ Michael R. Talcott, DVM, ${ }^{2}$ Daniel W. Moran, $\mathrm{PhD},{ }^{1}$ and \\ Eric C. Leuthardt, MD ${ }^{1,3}$
}

\begin{abstract}
1Department of Biomedical Engineering, and 2Division of Comparative Medicine, Washington University; and ${ }^{3}$ Department of Neurosurgery, Washington University School of Medicine, St. Louis, Missouri
\end{abstract}

\begin{abstract}
OBJECTIVE Instrumented spinal fusion continues to exhibit high failure rates in patients undergoing multilevel lumbar fusion or pseudarthrosis revision; with Grade II or higher spondylolisthesis; or in those possessing risk factors such as obesity, tobacco use, or metabolic disorders. Direct current (DC) electrical stimulation of bone growth represents a unique surgical adjunct in vertebral fusion procedures, yet existing spinal fusion stimulators are not optimized to enhance interbody fusion. To develop an advanced method of applying DC electrical stimulation to promote interbody fusion, a novel osteogenic spinal system capable of routing DC through rigid instrumentation and into the vertebral bodies was fabricated. A pilot study was designed to assess the feasibility of osteogenic instrumentation and compare the ability of osteogenic instrumentation to promote successful interbody fusion in vivo to standard spinal instrumentation with autograft.
\end{abstract}

METHODS Instrumented, single-level, posterior lumbar interbody fusion (PLIF) with autologous graft was performed at L4-5 in adult Toggenburg/Alpine goats, using both osteogenic spinal instrumentation (plus electrical stimulation) and standard spinal instrumentation (no electrical stimulation). At terminal time points ( 3 months, 6 months), animals were killed and lumbar spines were explanted for radiographic analysis using a SOMATOM Dual Source Definition CT Scanner and high-resolution Microcat II CT Scanner. Trabecular continuity, radiodensity within the fusion mass, and regional bone formation were examined to determine successful spinal fusion.

RESULTS Quantitative analysis of average bone density in pedicle screw beds confirmed that electroactive pedicle screws used in the osteogenic spinal system focally enhanced bone density in instrumented vertebral bodies. Qualitative and quantitative analysis of high-resolution CT scans of explanted lumbar spines further demonstrated that the osteogenic spinal system induced solid bony fusion across the L4-5 disc space as early as 6 weeks postoperatively. In comparison, inactive spinal instrumentation with autograft was unable to promote successful interbody fusion by 6 months postoperatively.

CONCLUSIONS Results of this study demonstrate that novel osteogenic spinal instrumentation supports interbody fusion through the focal delivery of DC electrical stimulation. With further technical development and scientific/clinical validation, osteogenic spinal instrumentation may offer a unique alternative to biological scaffolds and pharmaceutical adjuncts used in spinal fusion procedures.

http://thejns.org/doi/abs/10.3171/2016.1.SPINE13979

KEY WORDS osteogenic; lumbar; instrumentation; interbody fusion; direct current; electrical stimulation

$\mathrm{I}$ NJURIES of the back and spine represent the leading cause of lost productivity and immobility in the United States. ${ }^{24}$ Of the 279,000 surgeries conducted annually in the United States for the treatment of low-back pain, 160,000 involve such spinal fusion procedures. ${ }^{1,24}$ Recent literature suggests that only $68 \%$ of patients undergoing lumbar spinal fusion experienced satisfactory outcomes, and $20 \%-40 \%$ of spinal fusion procedures failed. ${ }^{30}$ Spinal instrumentation has been demonstrated to increase the rate of fusion in single-level lumbar interbody fusion, yet it exhibits high failure rates in patients undergoing multilevel lumbar fusion or pseudarthrosis revision; with Grade II or higher spondylolisthesis; or possessing risk factors such as obesity, tobacco use, or metabolic disorders. ${ }^{18,20,28,29,32}$ Exceedingly poor outcomes in "difficult to fuse" cases have led to an increased interest in novel surgical adjuncts capable of enhancing bone growth and fusion success following vertebral fixation.

ABBREVIATIONS AP = anteroposterior; $\mathrm{BMP}=$ bone morphogenetic protein; $\mathrm{DC}=$ direct current; $\mathrm{LR}=$ left-right.

SUBMITTED November 2, 2013. ACCEPTED January 21, 2016.

INCLUDE WHEN CITING Published online April 15, 2016; DOI: 10.3171/2016.1.SPINE13979. 
Direct current (DC) electrical stimulation of endogenous and/or exogenous bone matrix presents a promising method of enhancing osteogenesis in cases of spinal fusion. Prior studies demonstrate that damaged bone exhibits pronounced electronegativity, and that electrical potential serves as a critical cue in activating bone deposition and remodeling. ${ }^{10,13}$ Ensuing studies confirmed that "artificially" charging bone matrix through cathodic DC electrical stimulation induces local bone growth., ${ }^{2,9,12}$ Therapeutic application of DC electrical stimulation has been demonstrated in the healing of nonunion in the medial malleolus, ${ }^{11}$ and has been used to successfully induce bone formation at the site of lumbar spinal fusion. ${ }^{5}$ Numerous studies have demonstrated the safety and efficacy of DC electrical stimulation as a means of inducing bone growth and bony fusion in vivo, $3,6,14,15,22,29$ including several that have illustrated the potential of DC bone stimulation in human lumbar spinal fusion procedures. ${ }^{5,17,19,21,25,28}$

Existing implantable bone growth stimulators function by delivering a constant DC to coiled wire electrodes (cathodes) placed on or around lumbar vertebrae. Surgical placement of negatively charged wire electrodes between the transverse processes or posterior facets of adjacent vertebrae has been demonstrated to increase the mass, rate, and success of posterior and posterolateral bony fusion in various human and animal models. ${ }^{6,8,17,25}$ Yet existing spinal fusion stimulators do not enhance interbody fusion and are not approved for use in both posterior and anterior lumbar interbody fusion procedures. ${ }^{7,31}$ A significant opportunity, therefore, exists for the development of more effective methods of applying DC electrical stimulation to the lumbar spine for spinal fusion.

An integrated system of spinal instrumentation was developed to combine the mechanical advantages and ease of use of rigid spinal instrumentation with the osteoinductive capacity of DC electrical stimulation. The osteogenic system represents a modification of standard spinal rods and pedicle screws wherein cathodic DC is routed from an implantable stimulus generator (anode), through implanted metallic instrumentation, and focally delivered to the vertebral bodies through conductive portions of the threaded pedicle screw bodies (cathodes). This consolidated system of spinal instrumentation may offer a unique adjunct to existing methods of osteoinduction such as bone morphogenetic protein (BMP) scaffolds or fusion cages. ${ }^{16,27}$ This study was designed to examine the feasibility of the novel spinal system and provide preliminary evidence of the system's capability to stimulate successful interbody fusion in vivo in comparison with standard spinal instrumentation with autograft. To our knowledge, this report is the first description and in vivo demonstration of an integrated osteogenic system of spinal instrumentation involving electrical stimulation of bone growth through implanted pedicle screws.

\section{Methods}

\section{Experimental Design}

Skeletally mature, female Toggenburg/Alpine goats (Thomas Morris Inc.) were randomized into 6 experimental groups $(\mathrm{N}=6 ; \mathrm{n}=1$ goat in each group). Group I served as a negative control for operative technique, and the animal did not undergo surgical exposure of L-4 and L-5 vertebrae, discectomy, autograft placement, or implantation of spinal instrumentation. Groups II, III, and IV served to allow us to evaluate standard spinal instrumentation in animals undergoing surgical exposure of L-4 and L-5 vertebrae, discectomy, autograft placement, and implantation of inactive spinal instrumentation (no electrical stimulation) prior to euthanasia after 4 days, 3 months, and 6 months, respectively. Groups V and VI served to allow us to evaluate novel osteogenic spinal instrumentation in animals undergoing surgical exposure of L-4 and L-5 vertebrae, discectomy, autograft placement, and implantation of active spinal instrumentation (plus electrical stimulation) prior to euthanasia after 3 and 6 months, respectively. Lumbar spines and intact instrumentation were explanted to facilitate examination of pedicle screw placement on high-dose CT scans. Instrumentation was then removed from explanted spines prior to micro-CT scanning of bony fusion at the L4-5 disc space. Post hoc quantitative evaluation of micro-CT scans using custom image processing software was used to evaluate trabecular continuity and osteogenesis around the fusion site. All experimental procedures described complied with the Guide for the Care and Use of Laboratory Animals and were performed under the supervision of the Division of Comparative Medicine at Washington University.

\section{Design and Fabrication of Osteogenic Spinal Instrumentation}

Osteogenic instrumentation used in the present study consisted of conventional instrumentation modified to focally deliver low-level DC directly into the vertebral bodies. Instrumentation consisted of 3 components: a constant-current source (Fig. 1A), 1 pair of anodized titanium rods (Fig. 1B), and 2 pairs of selectively anodized pedicle screws (Fig. 1C). Constant current sources delivering 40 $\mu \mathrm{A}$ DC were a microcircuit board and battery (CR2032 lithium coin cell battery; Varta Microbattery Inc.) sealed in a stainless-steel housing. Titanium rods $(5.5-\mathrm{mm}$ diameter, $7.0-\mathrm{cm}$ length), tulips, and screws caps (based on Polaris spinal systems; Biomet Inc.) were custom-milled and

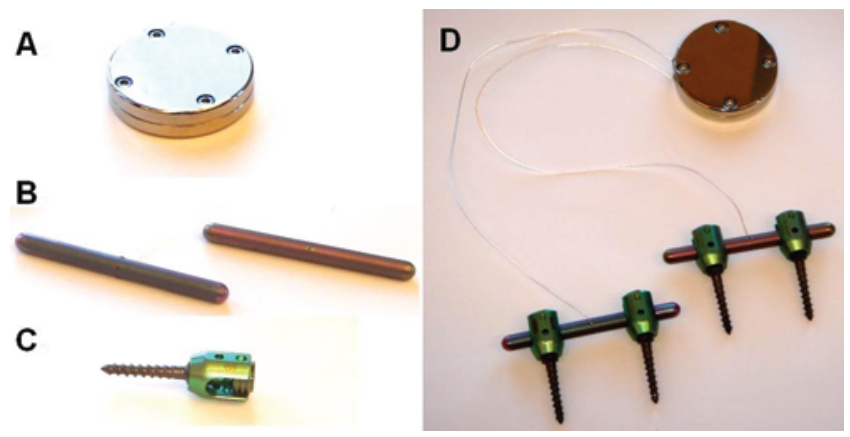

FIG. 1. Integrated spinal instrumentation used in the posterior lumbar interbody fusion procedure, including osteogenic pedicle screws. Instrumentation consisted of a custom-fabricated $40-\mu \mathrm{A}$ constant-current source (A), hard anodized titanium rods (B), and osteogenic pedicle screws (C). Assemblies facilitating delivery of DC to the conductive, threaded tips of attached pedicle screws were constructed in situ (D). 
hard-anodized to achieve a surface impedance of greater than $1 \mathrm{M} \Omega$, suitable to limit nonspecific current leakage into perispinal tissue. Custom segmental pedicle screws were prepared by selectively anodizing standard segmental pedicle screws (4-mm diameter, 25-mm long; Biomet Inc.). Threaded screw bodies were polished to achieve low surface impedance of less than $5 \Omega$. Selective anodization of pedicle screws enabled selective routing of DC through threaded screw bodies and into the vertebral body. Individual components were assembled intraoperatively to form a complete osteogenic spinal system (Fig. 1D).

\section{Surgical Procedure}

Animals underwent single-level lumbar discectomy and instrumented interbody fusion at L4 -5 through a posterior approach. General anesthesia was induced using isoflurane. L-4 and L-5 vertebrae were exposed through a $15-$ to $20-\mathrm{cm}$ midline incision followed by subperiosteal dissection of the musculature overlying the spinous processes, laminae, and apophyseal joints (Fig. 2A and B). The superior articular processes of L-5 and the spinous process of L-4 were then removed. Pilot holes were drilled into the pedicles under fluoroscopy and tamped prior to insertion of selectively anodized pedicle screws. A discectomy was then performed on the L4-5 intervertebral disc, and a standardized decorticated autologous bone graft, fashioned from the extracted spinous process, was inserted into the evacuated space (Fig. 2C and D). Anodized titanium rods attached to an implantable constant-current source were then inserted between the tulips of the 2 pedicle screws on the right side of the animal and secured. Animals in Groups III and IV received an inactive constant-current source, whereas animals in Groups V and VI received an active constant-current
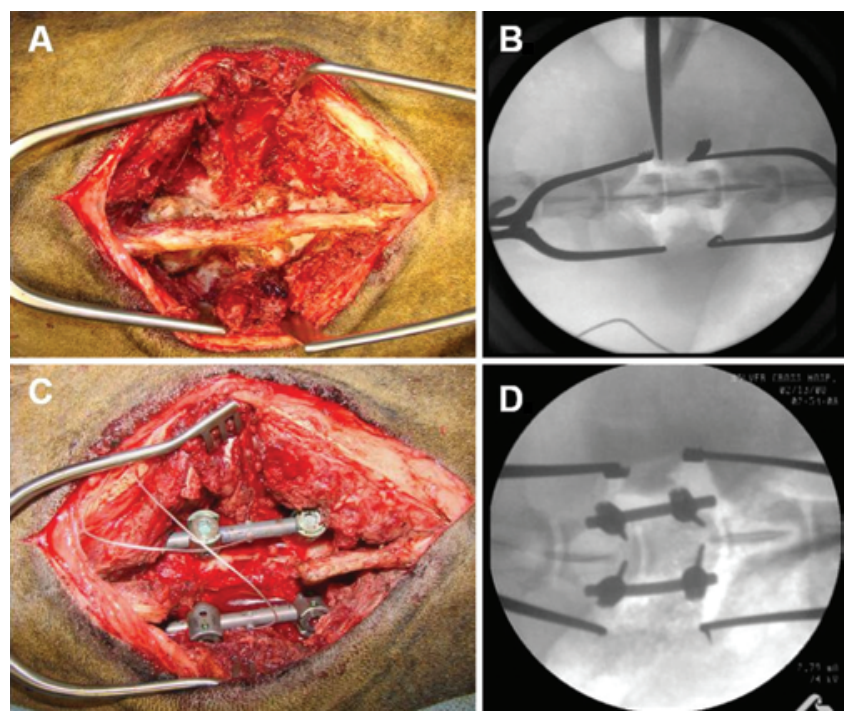

FIG. 2. Surgical placement of integrated osteogenic instrumentation in $\mathrm{L}-4$ and $\mathrm{L}-5$ vertebrae. Surgical dissection and isolation of the $\mathrm{L}-4$ and $\mathrm{L}-5$ vertebral bodies (A) was confirmed through identification of the L4-5 disc space (probe) under intraoperative fluoroscopy (B). Completed placement of experimental spinal instrumentation prior to closure (C). Intraoperative fluoroscopy additionally confirmed successful placement of osteogenic screws into the pedicles of L-4 and L-5 vertebrae (D). source. Constant current sources were implanted in the right paravertebral muscle bed near the caudal end of the primary incision and secured with 2-0 Vicryl. Placement of the completed spinal fusion system was documented via fluoroscopy (Fig. 3A and B). Musculature around the primary incision was then reapposed using 2-0 Vicryl, while the fascial layers were closed using 0 polydioxanone. Subcuticular, interrupted mattress sutures were placed using 2-0 Vicryl, and the skin was reapposed with surgical staples.

\section{Radiographic Analysis of Explanted Lumbar Spines}

At the terminal time point, animals were killed and lumbar spines were explanted en bloc with implanted in-
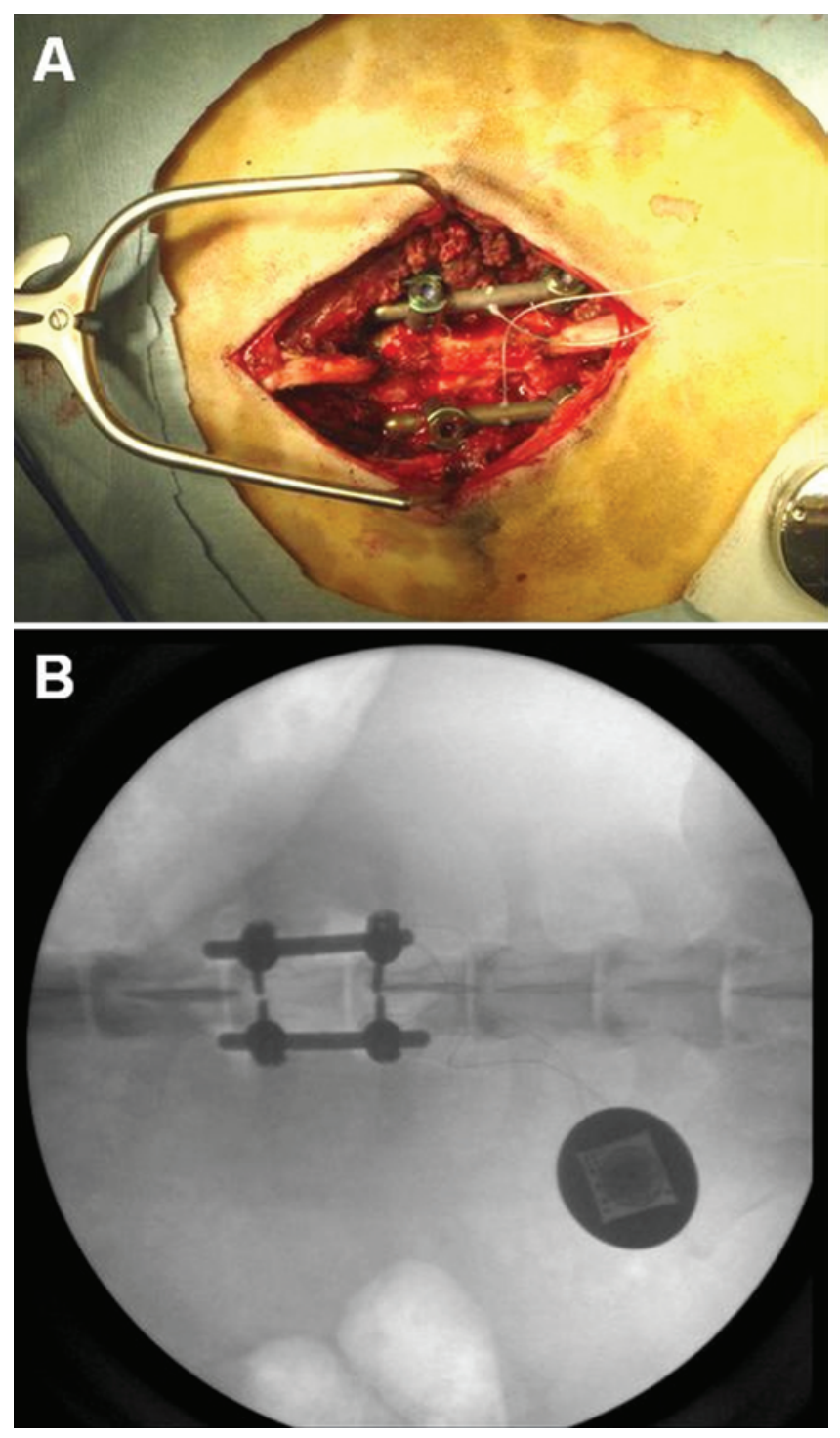

FIG. 3. Surgical placement of the constant-current source. Prior to implantation, constant-current sources were positioned to ensure a stressfree electrical connection to anodized rods (A). Stainless-steel constantcurrent sources were subcutaneously implanted $5 \mathrm{~cm}$ lateral and $5 \mathrm{~cm}$ caudal to implanted instrumentation. Radiographic appearance of spinal fusion instrumentation and attached constant-current source in vivo following closure (B). 
strumentation intact (Fig. 4A). Specimens were preserved in a solution of $10 \%$ neutral buffered formalin prior to radiographic evaluation, which typically lasted 24-72 hours. High-dose CT scans (U75u, $120 \mathrm{kVp}, 265 \mathrm{~mA}$ ) of whole specimens, including implanted instrumentation, were acquired using a SOMATOM Dual Source Definition CT Scanner (Siemens Healthcare) located in the Center for Clinical Imaging Research in the Mallinckrodt Institute of Radiology at Washington University School of Medicine. Acquired scans were reconstructed using InSpace software (Siemens Healthcare) to provide a highresolution $(300 \mu \mathrm{m}) 3 \mathrm{D}$ view of the relation between implanted instrumentation and lumbar vertebrae (Fig. 4B and $\mathrm{C}$ ).

High-resolution radiographic analysis of explanted lumbar spines also was conducted to assess bony fusion at the L4-5 disc space. High-resolution CT scans (bin of 1, $60 \mathrm{kVp}, 1,000 \mathrm{msec}, 500 \mu \mathrm{A}, \mathrm{n}=400$ views) of the L4-5 and L3-4 disc spaces were obtained using a Microcat II CT Scanner (Siemens Healthcare) present in the Washington University Small Animal Imaging Resource funded by the National Institutes of Health National Cancer Institute. Acquired scans were reconstructed using a 3D Feldkamp image reconstruction algorithm (spatial resolution $100 \mu \mathrm{m})$ and examined by an independent reviewer using Amira software (FEI Co.). Coronal and sagittal views and scans of reconstructed intervertebral segments were obtained to qualitatively assess the presence of bony fusion across the L4-5 disc space (Figs. 5-8).

\section{Quantitative Analysis of Trabecular Continuity in Acquired CT Scans}

Custom image-processing software was designed in Matlab (MathWorks Inc.) to quantify the degree of interbody fusion achieved by various types of spinal instru- mentation at terminal time points. Specifically, custom software estimated the average bone density at various intervals along a rostrocaudal axis extending through the L4-5 disc space (Fig. 9A). Examination of the spatial variability of average bone density provided a measure of trabecular continuity and uniform radiodensity within the fusion mass, both specific indicators of solid vertebral fusion. Average bone density versus rostrocaudal position within the disc space were normalized to the average bone density measured in the endogenous portion of L-4 vertebral bodies. Plots acquired through quantitative analysis of lumbar spines in various experimental groups were then compared to determine the effect of spinal instrumentation on success of spinal fusion (Fig. 9).

Custom image-processing software also was used to quantitatively examine focal osteogenesis surrounding implanted pedicle screws. Examination of the relationship between average bone density and distance from the pedicle screw hole provided a relative measure of focal osteoinduction surrounding the threaded screw body (Fig. 10A). Average bone density versus radial distance from the center of pedicle screw holes was normalized to the average bone density measured in evacuated pedicle screw holes. Plots acquired through quantitative analysis of lumbar spines were compared to determine the effect of spinal instrumentation on focal osteoinduction surrounding implanted pedicle screw bodies (Fig. 10).

\section{Results}

\section{Fabrication and Implantation of Osteogenic Spinal Systems}

Benchtop testing of assembled osteogenic spinal systems confirmed selective routing of DC through the threaded screw bodies of segmental pedicle screws and the housing of the constant-current source. Preoperative
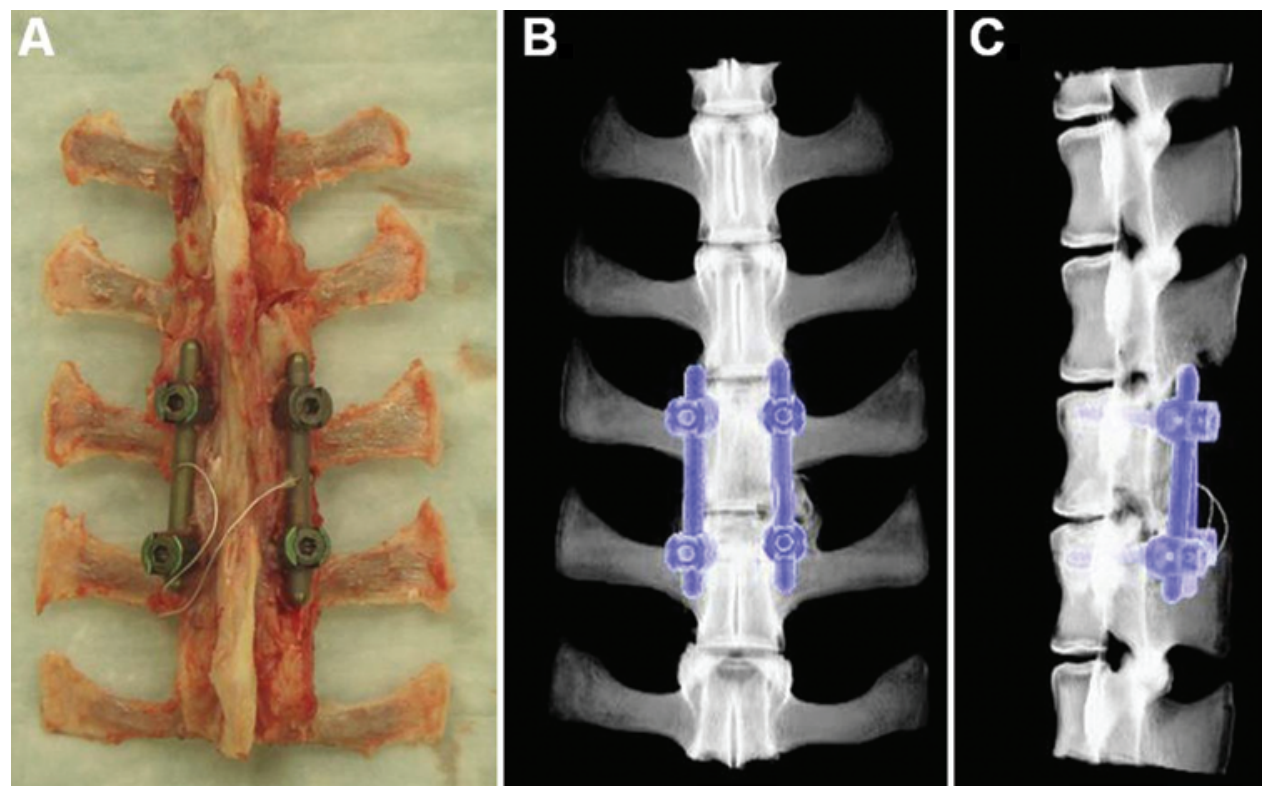

FIG. 4. Radiographic imaging of implanted instrumentation ex vivo. Explanted lumbar spines (A) were evaluated using high-dose CT scans. 3D reconstructions of CT scans evaluated in the posteroanterior (B) and lateral (C) planes confirmed proper localization and alignment of implanted instrumentation at terminal time points. 

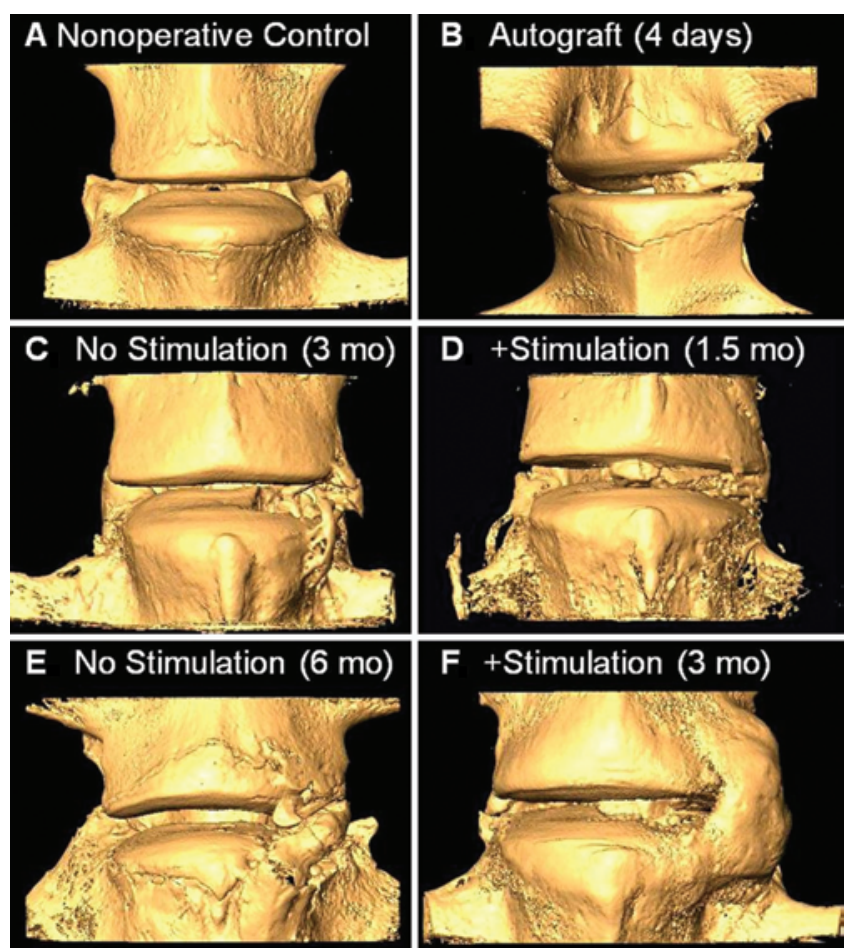

FIG. 5. Examination of the fusion mass at the $L 4-5$ disc space (coronal view). The L4-5 disc space was 3-dimensionally reconstructed using micro-CT scans. Nonoperative control (A) and 4-day postoperative (B) specimens demonstrate the normal appearance of the L4-5 disc space and the placement of the autologous bone graft, respectively. Specimens acquired from animals fitted with inactive instrumentation for 3 months (C) and 6 months (E) demonstrate minimal fusion mass and poor continuity between vertebrae. Specimens acquired from animals fitted with electrically active instrumentation for 1.5 months (D) and 3 months $(\mathbf{F})$ demonstrate success of fusion and increased fusion mass. + Stimulation $=$ with electrical stimulation.

assessment of assembled osteogenic spinal systems demonstrated that each system was functional and operative at the time of implantation. Active and inactive instrumentation was successfully implanted into the lumbar spine at the site of fusion. Long-term implantation of both spinal fusion systems was well tolerated by the animals, with no noticeable sequelae of the surgical procedure. All animals subsequently survived to predetermined terminal time points. The animal in Group VI was killed 6 weeks after surgery, because of complications associated with anesthesia.

\section{Macroscopic Evaluation of Explanted Lumbar Spines}

Gross examination of explanted lumbar spines revealed successful wound healing around both implanted spinal instrumentation and constant-current sources with no evidence of infection. 3D reconstructions of high-dose CT scans confirmed that all osteogenic and standard spinal systems were intact, secure, and properly aligned in the coronal and sagittal planes (Fig. 4A-C). Examination of high-dose CT scans further demonstrated that the threaded bodies of segmental screws were accurately placed in the pedicles of L-4 and L-5 vertebrae. Terminal testing of explanted power supplies further demonstrated that
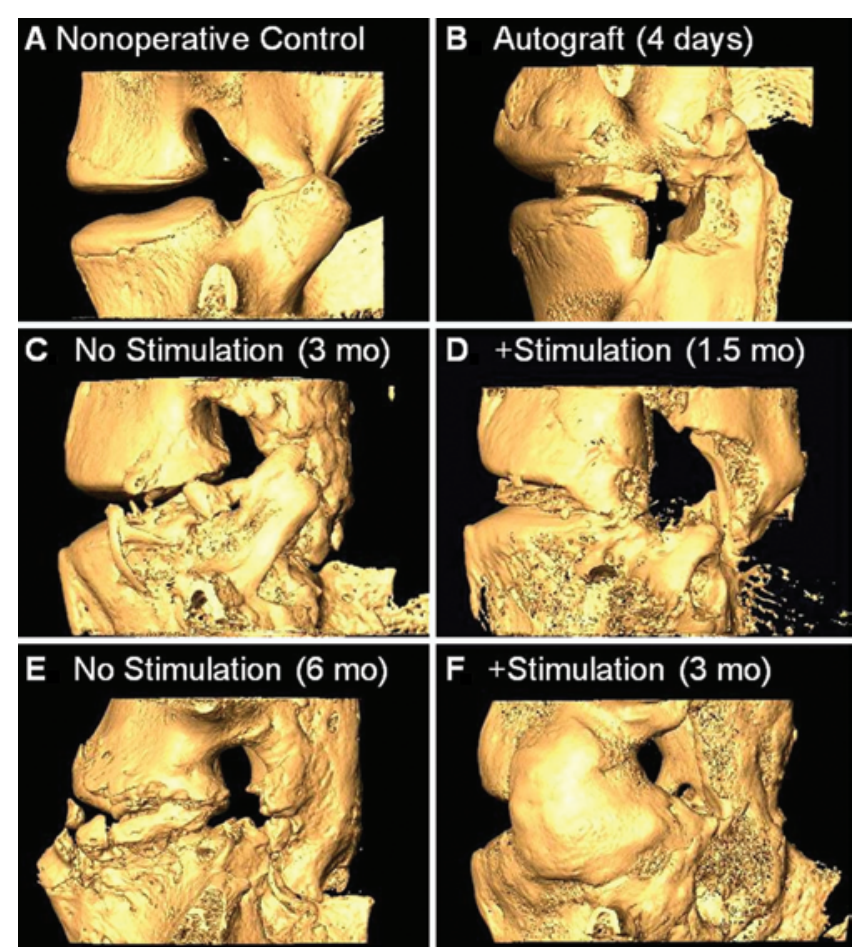

FIG. 6. Examination of fusion mass at $L 4-5$ disc space (sagittal view). L4-5 disc space was 3-dimensionally reconstructed using Micro-CT scans. Nonoperative control (A) and 4-day postoperative (B) specimens demonstrate the normal appearance of the L4-5 disc space and the placement of the autologous bone graft, respectively. Specimens acquired from animals fitted with inactive instrumentation for 3 months (C) and 6 months $(E)$ demonstrate a lack of successful fusion. Specimens acquired from animals fitted with electrically active instrumentation for 1.5 months $(\mathbf{D})$ and 3 months $(\mathbf{F})$ demonstrate increased fusion mass and increased continuity between vertebrae.

osteogenic spinal instrumentation remained functional throughout the duration of the study.

\section{Analysis of Interbody Fusion in Explanted Lumbar Spines: 3D Reconstruction of Micro-CT Scans}

$3 \mathrm{D}$ reconstructions of micro-CT scans demonstrated increased fusion mass and increased success of fusion in lumbar spines implanted with the osteogenic spinal system. Reconstructions obtained from nonoperative (Figs. 5A and 6A) and operative disc spaces (Figs. 5B and 6B) demonstrated successful insertion of autologous bone graft into the L4-5 disc space and negligible anatomical effect of instrumentation at the site of fusion. Reconstructions obtained from spines instrumented with the standard spinal system (no electrical stimulation) for 3 months (Figs. 5C and 6C) and 6 months (Figs. 5E and 6E) demonstrated positive signs of bone remodeling yet little fusion mass in the L4-5 disc space and no bony bridging of the vertebral bodies (Figs. 6C and E). In contrast, reconstructions obtained from spines instrumented with osteogenic instrumentation (plus electrical stimulation) for 1.5 months (Figs. 5D and 6D) and 3 months (Figs. 5F and 6F) demonstrated positive signs of bone remodeling as well as increased fusion mass in the L4-5 disc space and solid bridging of the vertebral bodies. 

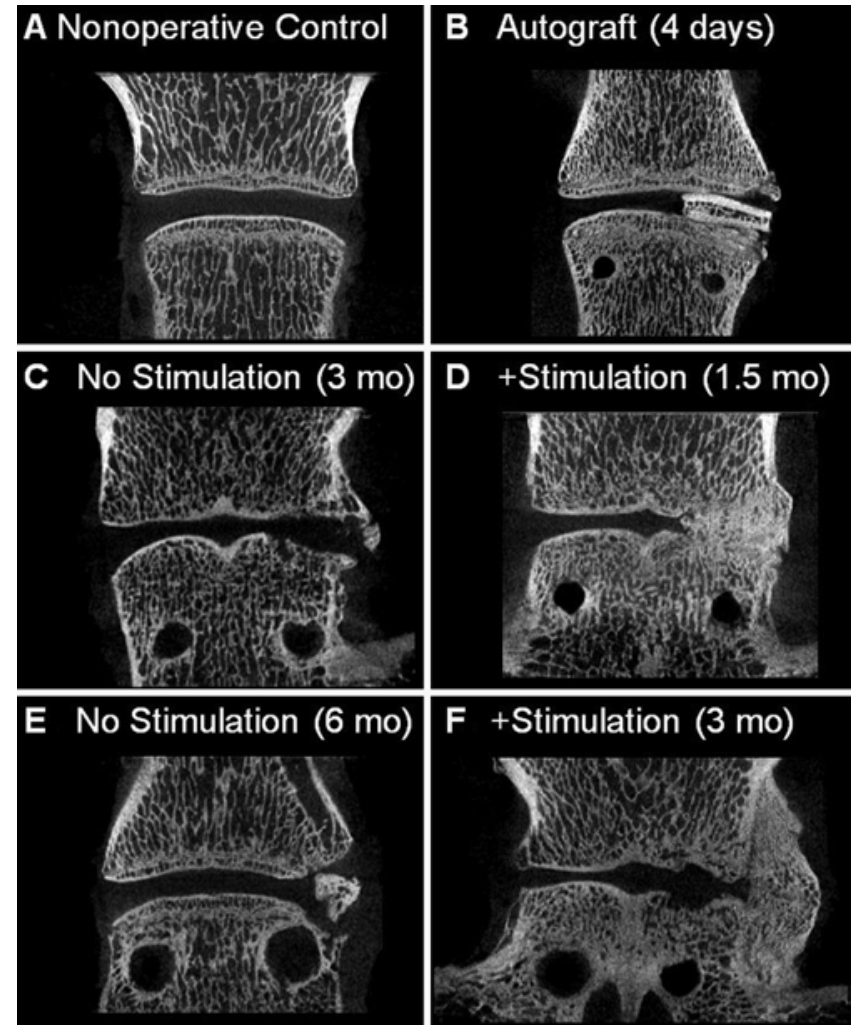

FIG. 7. Radiographic examination of bony fusion at the L4-5 disc space (coronal view). Micro-CT scans of nonoperative control (A) and 4-day postoperative (B) specimens demonstrate the normal appearance of the L4-5 disc space and the placement of the autologous bone graft, respectively. Specimens acquired from animals fitted with inactive instrumentation for 3 months $(\mathbf{C})$ and 6 months $(\mathbf{E})$ demonstrate discontinuity between adjacent vertebral bodies. Specimens acquired from animals fitted with electrically active instrumentation for 1.5 months (D) and 3 months $(\mathbf{F})$ demonstrate solid fusion between adjacent vertebral bodies.

3D reconstructions further demonstrated increased bone deposition at the site of fusion and increased preservation of autologous bone graft material in the presence of osteogenic spinal instrumentation. Detailed analysis of bony tissue present in the L4-5 disc space revealed a net loss in mineralized bone matrix in the presence of inactive spinal instrumentation (Fig. 5C) and a net gain in mineralized bone matrix in the presence of an osteogenic spinal instrumentation (Fig. 5F). This observation suggests enhanced bone deposition in the presence of electroactive spinal system consistent with prior demonstrations of the osteoinductive effect of DC electrical stimulation. ${ }^{3,6,14,22,29}$ Further examination of 3D reconstructions revealed that autologous bone graft material was better preserved in spines instrumented with osteogenic spinal systems (Figs. 5D and 6D) than in those instrumented with standard spinal systems (Figs. 5C and 6C). Evidence of bone remodeling and bone deposition in all specimens was focally observed around implanted autologous bone grafts.

\section{Qualitative Analysis of Interbody Fusion in Explanted Lumbar Spines: Single Micro-CT Slices}

Examination of micro-CT slices confirmed induction of solid bony fusion in the presence of osteogenic spinal
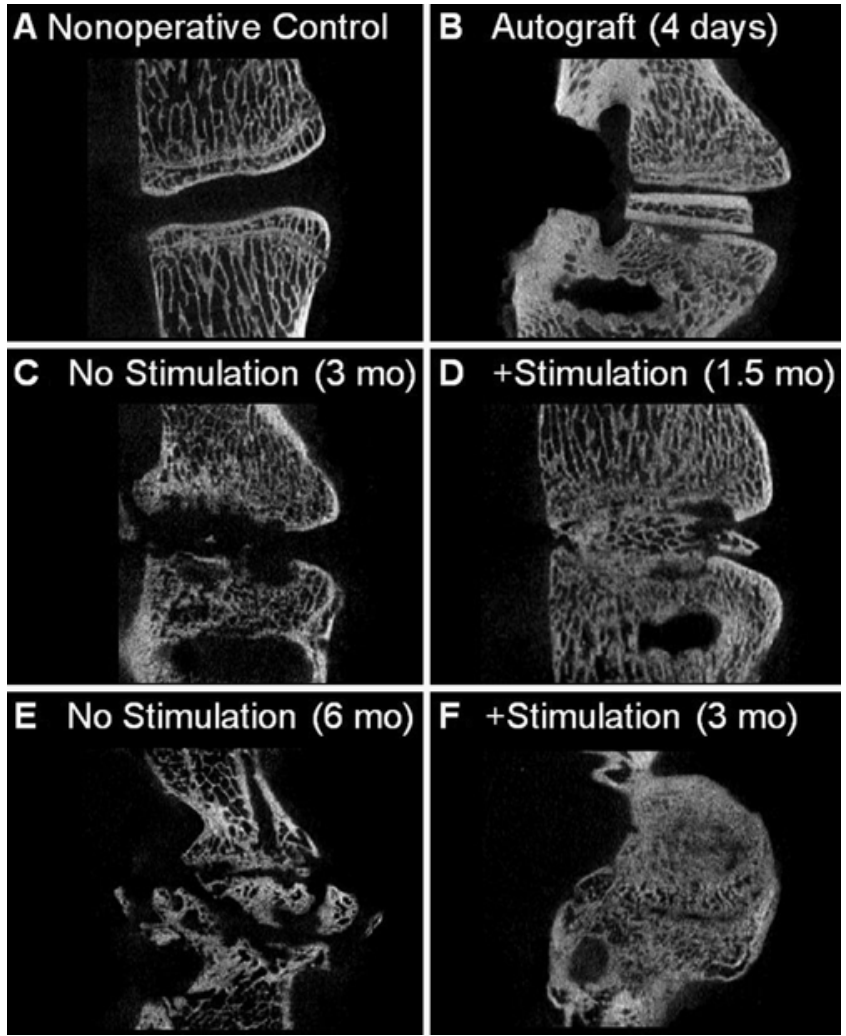

FIG. 8. Radiographic examination of bony fusion at the L4-5 disc space (sagittal view). Micro-CT scans of nonoperative control (A) and 4-day postoperative (B) specimens demonstrate the normal appearance of the L4-5 disc space and the placement of the autologous bone graft, respectively. Specimens acquired from animals fitted with inactive instrumentation for 3 months (C) and 6 months (E) demonstrate a lack of bony fusion between adjacent vertebral bodies. Specimens acquired from animals fitted with electrically active instrumentation for 1.5 months (D) and 3 months (F) demonstrate increased continuity between adjacent vertebral bodies.

instrumentation. Examination of scans obtained from spines instrumented with standard spinal hardware for 3 months (Figs. 7C and 8C) and 6 months (Figs. 7E and 8E) demonstrated significant resorption of the bone graft and discontinuity between L-4 and L-5 vertebrae. In contrast, spines instrumented with osteogenic spinal systems for 1.5 months (Figs. 7D and 8D) and 3 months (Figs. 7F and 8F) demonstrated mineralized fusion masses within the L4-5 disc space. Despite variability in the mediolateral position of the fusion mass, possibly caused by variation position of implanted bone grafts, all fusion masses successfully bridged L-4 and L-5 vertebrae. Detailed analysis of fusion masses demonstrated continuous regions of woven and trabecular bone, trabecular bridging with vertebral bodies, and consistent radiodensity across the intervertebral disc space.

Micro-CT slices additionally demonstrated local enhancement of bone deposition around implanted osteogenic pedicle screws. Comparison of micro-CT scans obtained from spines instrumented with a standard spinal system for 3 months (Figs. 7C and 8C) and an osteogenic spinal system for 1.5 months (Figs. 7D and 8D) revealed increased bone density surrounding pedicle screw beds 

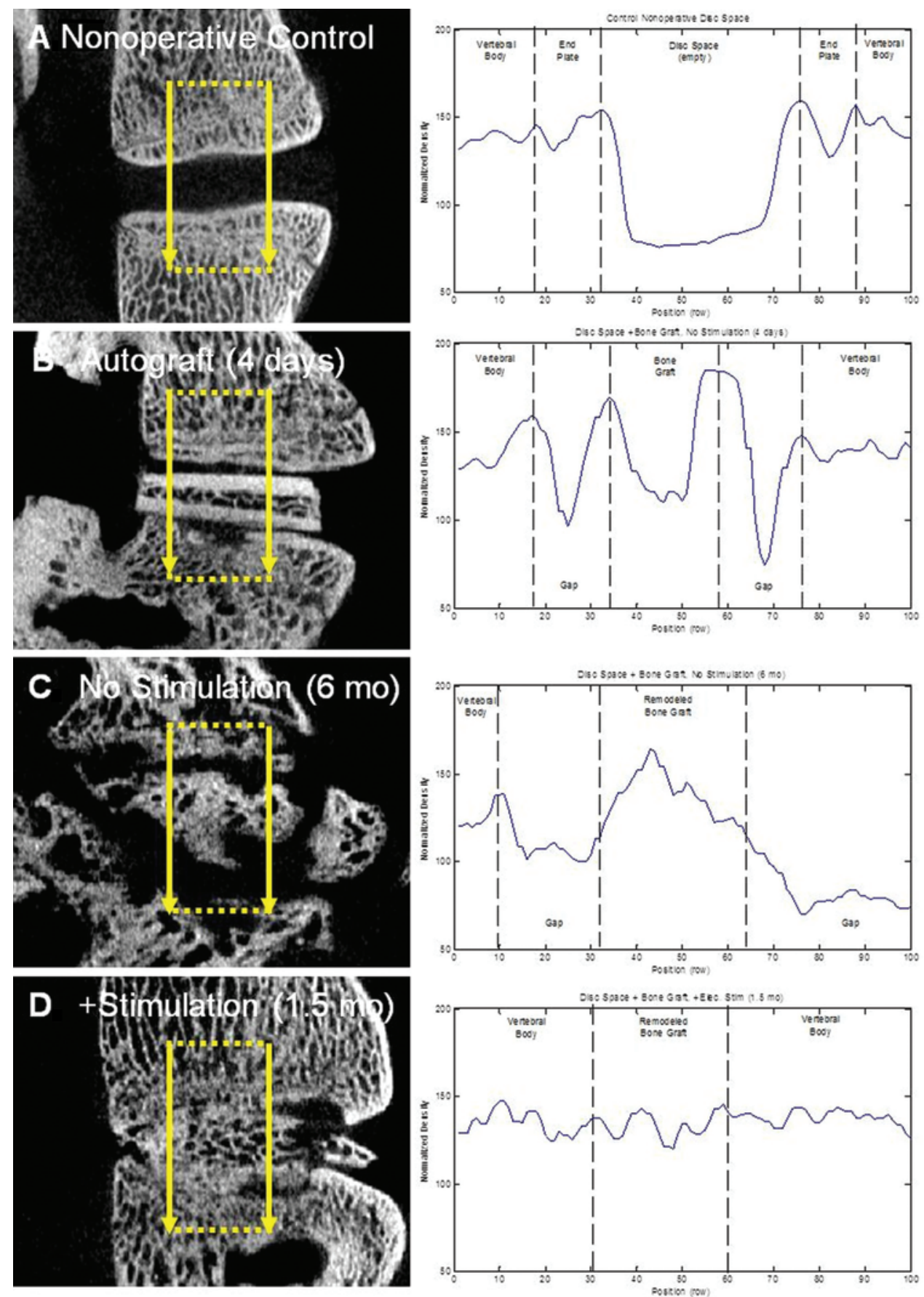

FIG. 9. Quantitative analysis of trabecular continuity across the L4-5 disc space. Analysis of selected regions of sagittal microCT scans (yellow boxes) using custom-designed software quantified the presence of bony tissue at various points through the L4-5 disc space. Assessment of nonoperative control (A) scans demonstrate absence of bony tissue in the disc space, represented by the nadir in the corresponding plot. Examination scans acquired from a 4-day postoperative specimen (B) demonstrate pronounced dense cortices and perigraft gaps, represented by large maxima and minima, signifying an absence of bony fusion. Scans acquired from an animal fitted with inactive instrumentation for 6 months demonstrate similar perigraft gaps (C), whereas scans acquired from an animal fitted with active instrumentation for 1.5 months demonstrate positive trabecular continuity and graft incorporation signifying successful bony fusion (D). +Elec = electrical stimulation.

containing electroactive pedicle screws. Increased bone deposition may result from higher current densities at the bone-screw interface, consistent with prior studies demonstrating a positive correlation between DC density and osteogenic activity. ${ }^{4,6,9}$

\section{Quantitative Analysis of Interbody Fusion in Explanted Lumbar Spines}

Quantitative analysis of average bone density across the L4-5 disc space confirmed successful interbody fusion in spines instrumented with osteogenic spinal sys- 

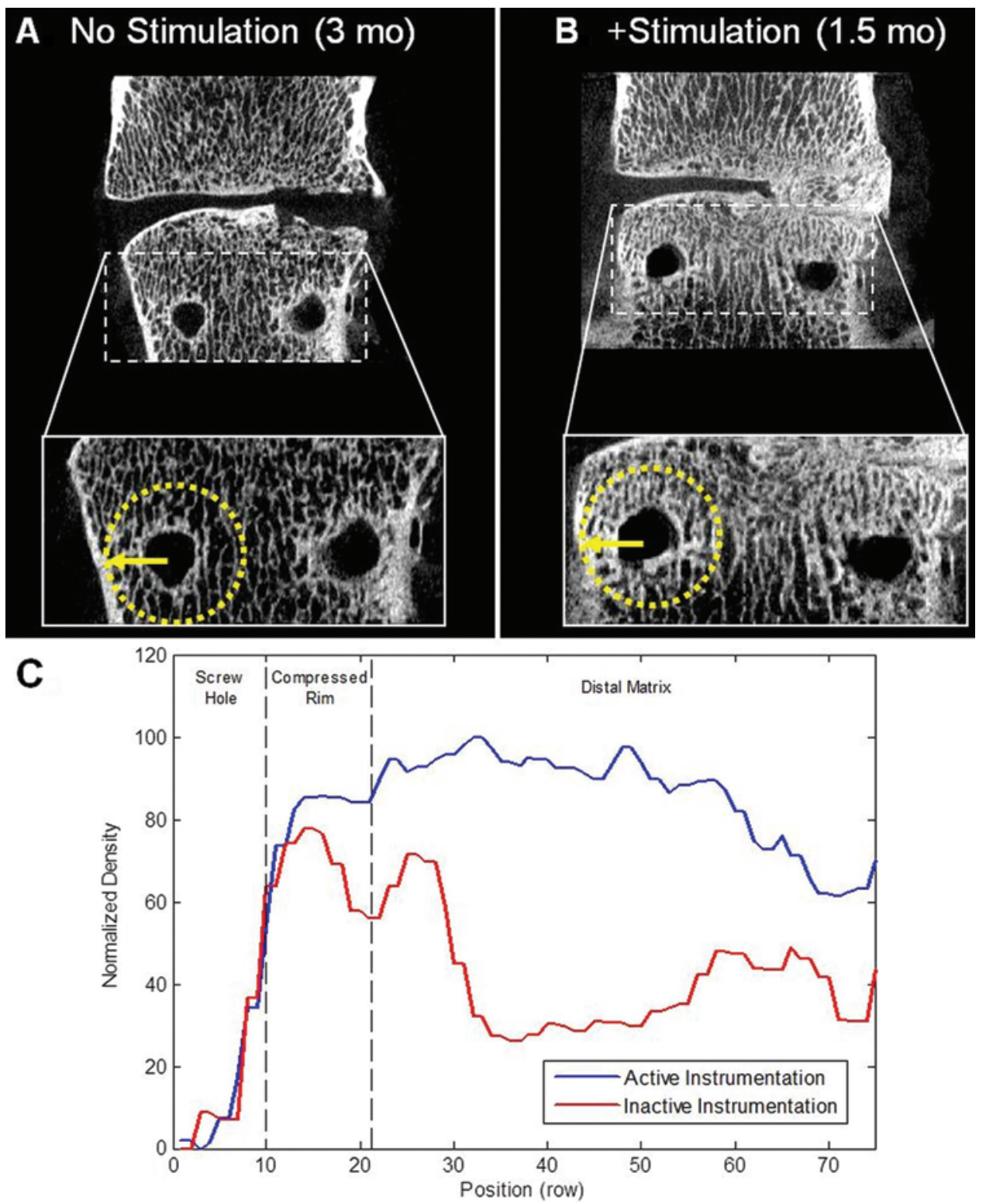

FIG. 10. Quantitative analysis of bone density surrounding pedicle screw beds. Comparison of coronal micro-CT scans obtained from animals fitted with inactive instrumentation for 3 months (A) and active instrumentation for 1.5 months (B) demonstrates increased bone density surrounding electroactive pedicle screws. Custom software designed to quantify average bone density extending radially from the center of pedicle screw beds was used to examine selected regions of the micro-CT scans (yellow circles). Resulting plots $(\mathbf{C})$ demonstrate increased bone density beyond the compacted rim of pedicle screw beds once containing electroactive pedicle screws.

tems. Quantitative analysis demonstrated significant variability in bone density and discontinuity across the L4-5 disc space in spines instrumented with a standard spinal system for 6 months (Fig. 9C). Evaluation of scans obtained from spines instrumented with an osteogenic spinal system for 1.5 months demonstrated reduced spatial variability in bone density measurements across the target disc space (Fig. 9D). The absence of dramatic minima in bone density plots confirms trabecular continuity between adjacent vertebral bodies and suggests successful graft incorporation in the present of osteogenic instrumentation.

Quantitative analysis of average bone density surround- ing pedicle screw beds further confirmed focal enhancement of bone density in the vertebral bodies instrumented with osteogenic pedicle screws. Evaluation of pedicle screw beds in L-5 vertebrae following implantation of standard spinal instrumentation for 3 months (Fig. 10A) and osteogenic spinal instrumentation for 1.5 months (Fig. 10B) demonstrated significant differences in bone density surrounding implanted pedicle screws (Fig. 10C). Both types of pedicle screw induced a high-density rim of compacted bone as a result of instrumentation, yet electroactive pedicle screws were observed to induce higher average bone density beyond the compacted rim compared with standard pedicle screws. 


\section{Discussion}

The present study represents the first in vivo trial of an osteogenic spinal system capable of routing DC electrical stimulation through rigid instrumentation to the site of fusion. Although multiple studies have demonstrated simultaneous application of rigid instrumentation and bone growth stimulators in spinal fusion procedures, ${ }^{23,26}$ few have demonstrated successful interbody fusion as a direct result of electrical stimulation. Inability to achieve interbody fusion with existing bone-growth stimulators is primarily due to limitations in the peripheral placement of stimulator leads and hardware. ${ }^{19,25}$ Integration of bonegrowth stimulation technology directly into standard rigid instrumentation, as outlined in this study, was performed to eliminate hardware incompatibility and improve focal delivery of DC electrical stimulation to the fusion site.

Preliminary testing in a large-animal model of instrumented posterior lumbar interbody fusion provides initial confirmation of the feasibility of our system of osteogenic instrumentation. Osteogenic instrumentation was noted to enhance the deposition of new bone within the target disc space, focally increase bone density within the vertebral body, and support successful fusion as early as 6 weeks postoperatively. Observation of solid fusion 6 weeks after implantation in the goat spine suggests that our electroactive system may provide relatively rapid fusion in a difficult-to-fuse model. Prior studies examining the use of fusion cages and spinal fusion stimulators in sheep and dogs do not report successful fusion until 3 or 4 months postoperatively. ${ }^{3,6,14,29}$ Together, these results provide preliminary evidence that osteogenic instrumentation supplying DC directly to the vertebral bodies may enhance interbody fusion in vivo even in difficult-to-fuse scenarios.

This study further suggests that integrated osteogenic spinal instrumentation may offer a unique alternative to existing osteoinductive strategies. Given the nonpharmacological nature of DC electrical stimulation, osteogenic instrumentation may offer increased temporal and spatial control of new bone formation at the fusion site. Osteogenic instrumentation, therefore, may offer a future alternative to biological implants and BMP scaffolds. Future investigations will investigate the mechanism by which integrated osteogenic instrumentation induces a proregenerative anabolic microenvironment and will explicitly examine the clinical efficacy of osteogenic spinal instrumentation relative to existing biological technologies.

Although this study offers, to our knowledge, a first proof of principle of integrated osteogenic spinal hardware, it is important to note the limitations of the study design. As a pilot study, this work leveraged few animal subjects per cohort. Thus, although notable differences were observed between the stimulated and nonstimulated spines, the statistical strength of these differences cannot be asserted. The spinal biomechanics in a tetrapod large-animal model also differ significantly from those observed in humans. Furthermore, it is important to note that the design of osteogenic instrumentation reported in this study does not represent an ideal clinical form factor and carries technical inefficiencies associated with the use and placement of an implantable power supply. Finally, it is important to note that variability was observed in the location of bone deposition around implanted osteogenic instrumentation. Bone formation at the lateral edge of target intervertebral spaces was most likely attributed to heterogeneity in DC passing through the bony tissue and possibly due to movement of autograft (Fig. 7F).

Despite these limitations, this study supports continued investigations into the clinical efficacy and utility of integrated osteogenic spinal instrumentation. Future studies will examine means of optimizing the design and usability of osteogenic instrumentation and of eliminating the need for an implantable power source. More definitive assertions of the efficacy of osteogenic instrumentation will also be achieved via suitably powered studies comparing the success rate of the osteogenic spinal system to existing surgical adjuncts, such as fusion cages and BMP-loaded scaffolds.

\section{Conclusions}

This study highlights the feasibility of osteogenic spinal instrumentation capable of routing DC through rigid instrumentation into the vertebral bodies to promote interbody fusion. In vivo demonstration that osteogenic instrumentation supports successful fusion suggests the system may present a unique alternative to existing adjuncts used in spinal fusion surgery. Results of this study support further investigation into the clinical efficacy of integrated osteogenic instrumentation.

\section{Acknowledgments}

This study was funded by a Bear Cub Translational Grant awarded by Washington University in St. Louis.

We thank Arnold Heidbreder, Erik Zellmer, and the veterinary staff in the Division of Comparative Medicine at Washington University School of Medicine for valuable assistance throughout this study.

\section{References}

1. Andersson GB: Epidemiological features of chronic low-back pain. Lancet 354:581-585, 1999

2. Bassett CA, Pawluk RJ, Becker RO: Effects of electrical currents on bone in vivo. Nature 204:652-654, 1964

3. Bozic KJ, Glazer PA, Zurakowski D, Simon BJ, Lipson SJ, Hayes WC: In vivo evaluation of coralline hydroxyapatite and direct current electrical stimulation in lumbar spinal fusion. Spine (Phila Pa 1976) 24:2127-2133, 1999

4. Brighton CT, Friedenberg ZB, Black J, Esterhai JL Jr, Mitchell JE, Montique F Jr: Electrically induced osteogenesis: relationship between charge, current density, and the amount of bone formed: introduction of a new cathode concept. Clin Orthop Relat Res (161):122-132, 1981

5. Dwyer AF, Wickham GG: Direct current stimulation in spinal fusion. Med J Aust 1:73-75, 1974

6. France JC, Norman TL, Santrock RD, McGrath B, Simon BJ: The efficacy of direct current stimulation for lumbar intertransverse process fusions in an animal model. Spine (Phila Pa 1976) 26:1002-1008, 2001

7. Fraser RD: Interbody, posterior, and combined lumbar fusions. Spine (Phila Pa 1976) 20 (24 Suppl):167S-177S, 1995

8. Fredericks DC, Smucker J, Petersen EB, Bobst JA, Gan JC, Simon BJ, et al: Effects of direct current electrical stimulation on gene expression of osteopromotive factors in a posterolateral spinal fusion model. Spine (Phila Pa 1976) 32:174-181, 2007 
9. Friedenberg ZB, Andrews ET, Smolenski BI, Pearl BW, Brighton CT: Bone reaction to varying amounts of direct current. Surg Gynecol Obstet 131:894-899, 1970

10. Friedenberg ZB, Brighton CT: Bioelectric potentials in bone. J Bone Joint Surg Am 48:915-923, 1966

11. Friedenberg ZB, Harlow MC, Brighton CT: Healing of nonunion of the medial malleolus by means of direct current: a case report. J Trauma 11:883-885, 1971

12. Friedenberg ZB, Zemsky LM, Pollis RP, Brighton CT: The response of non-traumatized bone to direct current. J Bone Joint Surg Am 56:1023-1030, 1974

13. Fukada E, Yasuda I: On the piezoelectric effect of bone. J Phys Soc Jpn 12:1158-1162, 1957

14. Kahanovitz N, Arnoczky SP: The efficacy of direct current electrical stimulation to enhance canine spinal fusions. Clin Orthop Relat Res (251):295-299, 1990

15. Kahanovitz N, Pashos C: The role of implantable direct current stimulation in the critical pathway for lumbar spinal fusion. J Case Manag 2:2-8, 1996

16. Kahanovitz N: Spine update. The use of adjunctive electrical stimulation to enhance the healing of spine fusions. Spine (Phila Pa 1976) 21:2523-2525, 1996

17. Kane WJ: Direct current electrical bone growth stimulation for spinal fusion. Spine (Phila Pa 1976) 13:363-365, 1988

18. Kornblatt MD, Casey MP, Jacobs RR: Internal fixation in lumbar spine fusion. Clin Orthop Relat Res (203):141-150, 1986

19. Kucharzyk DW: A controlled prospective outcome study of implantable electrical stimulation with spinal instrumentation in a high-risk spinal fusion population. Spine (Phila Pa 1976) 24:465-469, 1999

20. Lorenz M, Zindrick M, Schwaegler P, Vrbos L, Collatz MA, Behal R, et al: A comparison of single-level fusions with and without hardware. Spine (Phila Pa 1976) 16 (8 Suppl):S455-S458, 1991

21. Meril AJ: Direct current stimulation of allograft in anterior and posterior lumbar interbody fusions. Spine (Phila Pa 1976) 19:2393-2398, 1994

22. Nerubay J, Marganit B, Bubis JJ, Tadmor A, Katznelson A: Stimulation of bone formation by electrical current on spinal fusion. Spine (Phila Pa 1976) 11:167-169, 1986

23. Oishi M, Onesti ST: Electrical bone graft stimulation for spinal fusion: a review. Neurosurgery 47:1041-1056, 2000

24. Praemer A, Furner S, Rice DP: Musculoskeletal Conditions in the United States. Park Ridge, IL: American Academy of Orthopedic Surgeons, 1999

25. Rogozinski A, Rogozinski C: Efficacy of implanted bone growth stimulation in instrumented lumbosacral spinal fusion. Spine (Phila Pa 1976) 21:2479-2483, 1996

26. Sidhu KS, Herkowitz HN: Spinal instrumentation in the man- agement of degenerative disorders of the lumbar spine. Clin Orthop Relat Res 335:39-53, 1997

27. Smoljanovic T, Bojanic I, Delimar D: Adverse effects of posterior lumbar interbody fusion using rhBMP-2. Eur Spine J 18:920-924, 2009 (Letter)

28. Tejano NA, Puno R, Ignacio JM: The use of implantable direct current stimulation in multilevel spinal fusion without instrumentation. A prospective clinical and radiographic evaluation with long-term follow-up. Spine (Phila Pa 1976) 21:1904-1908, 1996

29. Toth JM, Seim HB III, Schwardt JD, Humphrey WB, Wallskog JA, Turner AS: Direct current electrical stimulation increases the fusion rate of spinal fusion cages. Spine (Phila Pa 1976) 25:2580-2587, 2000

30. Turner JA, Ersek M, Herron L, Haselkorn J, Kent D, Ciol MA, et al: Patient outcomes after lumbar spinal fusions. JAMA 268:907-911, 1992

31. Wang JC, Mummaneni PV, Haid RW: Current treatment strategies for the painful lumbar motion segment: posterolateral fusion versus interbody fusion. Spine (Phila Pa 1976) 30 (16 Suppl):S33-S43, 2005

32. Zdeblick TA: A prospective, randomized study of lumbar fusion. Preliminary results. Spine (Phila Pa 1976) 18:983-991, 1993

\section{Disclosures}

Dr. MacEwan holds ownership interests in OsteoVantage, Inc., Acera Surgical, Inc., and Retectix, LLC, and has served as a consultant for Biogenerator. Dr. Moran holds ownership interests in OsteoVantage, Inc. Dr. Leuthardt holds patent and/or ownership interests in OsteoVantage, Inc., Neurolutions, Acera Surgical, Inc., Pear Therapeutics, and General Sensing.

\section{Author Contributions}

Conception and design: all authors. Acquisition of data: MacEwan, Talcott. Analysis and interpretation of data: Leuthardt, MacEwan, Moran. Drafting the article: MacEwan. Critically revising the article: Leuthardt, MacEwan. Reviewed submitted version of manuscript: all authors. Approved the final version of the manuscript on behalf of all authors: Leuthardt. Administrative/technical/material support: MacEwan. Study supervision: MacEwan, Talcott

\section{Correspondence}

Eric C. Leuthardt, Department of Neurosurgery, Washington University, St. Louis, Campus Box 8057, 660 S Euclid Ave., St. Louis, MO 63110.email: leuthardte@wudosis.wustl.edu. 